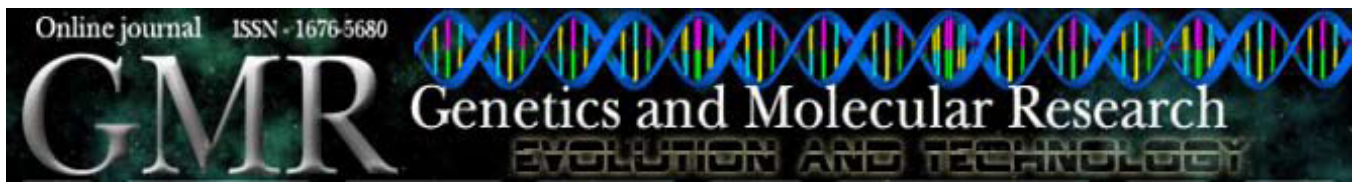

\title{
Expression patterns of the STAG gene in intact and regenerating planarians (Dugesia japonica)
}

\author{
Z.Q. Yuan, B.S. Zhao and J.Y. Zhang \\ Laboratory of Developmental and Evolutionary Biology, \\ School of Life Sciences, Shandong University of Technology, \\ Zibo, P.R. China \\ Corresponding author: B.S. Zhao \\ E-mail: zhaobosheng@sdut.edu.cn
}

Genet. Mol. Res. 10 (1): 410-418 (2011)

Received August 30, 2010

Accepted January 8, 2011

Published March 9, 2011

DOI 10.4238/vol10-1gmr1042

\begin{abstract}
We examined the spatial and temporal expression of the planarian Dugesia japonica STAG-related gene (DjStag), in both intact and regenerating planarians, by whole-mount in situ hybridization and relative quantitative real-time PCR. The first localized transcripts of DjStag were detected in the blastemas three days after amputation, in all regenerates including those from head, tail and trunk pieces. The maximum level of expression of DjStag transcripts occurred at five days after cutting. After regeneration for seven days, DjStag was weakly expressed. A similar decrease occurs regardless of the orientation of the cut. The expression pattern did not differ significantly in the different types of regeneration. Relative quantitative real-time PCR analysis of DjStag mRNA indicated that the expression of DjStag mRNA was increased after amputation compared to that in normal intact planarians, and the maximum level of expression of DjStag transcripts occurred at five days after amputation. All results suggest that DjStag, implicated in planarian
\end{abstract}


regeneration, plays a role in maintaining the ability of pluripotent stem cells to regenerate lost tissue in planarians.

Key words: Planarian; Regeneration; STAG; Gene expression; In situ hybridization

\section{INTRODUCTION}

In eukaryotes, the two copies of chromosomes are generated in the S-phase. Sister chromatids remain connected until they are separated in anaphase of mitosis. Sister chromatid cohesion depends on cohesin, a protein complex that is highly conserved in evolution and consists of at least four subunits: two structural maintenance chromosome proteins, Smc1 and Smc3, and two sister chromatid cohesion proteins, Scc1 and Scc3 (Michaelis et al., 1997; Losada et al., 1998; Sumara et al., 2000; Haering and Nasmyth, 2003). Cohesins form a ring around the sister DNA molecules, with Smc1 and Smc3 forming a V-shaped heterodimer bridged by Scc1 (Anderson et al., 2002; Gruber et al., 2003). Scc3 binds to the complex by associating with the C-terminal domain of Scc1 (Gruber et al., 2003). Cells of humans, hoptoad and other higher eukaryotes contain two mitotic orthologs of Scc3, called STAG1/SA1 and STAG2/SA2. Cohesin complexes in these cells contain either SA1 or SA2, but not both (Losada et al., 2000; Pezzi et al., 2000; Sumara et al., 2000; Prieto et al., 2001; Krasikova et al., 2005).

Although previous study on cohesins has been mainly focused on chromatid cohesion and segregation, certain cohesins have been implicated in different cellular processes (Lara-Pezzi et al., 2004). Caspase proteolysis of Scc1 can be observed in cells undergoing apoptosis in response to diverse stimuli, preceding chromatin condensation and amplifying the death signal (Chen et al., 2002; Pati et al., 2002). Cohesin function is also essential for DNA repair (Sjogren and Nasmyth, 2001). The possible function of STAG, however, is not completely understood. Lara-Pezzi et al. (2004) provide evidence suggesting that STAG functions as a transcriptional co-activator by a mechanism involving protein-protein interactions with transcription factors (Losada et al., 2000).

Planarians (Platyhelminthes, Tricladida) are excellent models for the study of the biology of regeneration because of their remarkable ability to rebuild a complete individual from any small amputated fragment of their body (Salvetti et al., 1998; Reddien and Sanchez, 2004). However, little is known about the STAG gene in this important animal. In this report, we show the spatial and temporal distribution of the planarian Dugesia japonica STAG-related gene (DjStag) (GenBank accession No. GQ503880) in intact and regenerating planarians as revealed by in situ hybridization and relative quantitative realtime polymerase chain reaction (PCR).

\section{MATERIAL AND METHODS}

\section{Animals and culture conditions}

The planarians used belong to the race of D. japonica collected from a fountain in Quanhetou, Boshan, China. The animals were kept in autoclaved tap water at $20^{\circ} \mathrm{C}$. Planar- 
ians had been starved for at least 1 week before the experiments. Some planarians were cut both anterior and posterior to the pharynx with a razor blade and each piece was allowed to regenerate at $20^{\circ} \mathrm{C}$.

\section{Whole-mount in situ hybridization}

Whole-mount in situ hybridization was performed on intact and regenerating planarians essentially as described previously (Reddien et al., 2007; Palakodeti et al., 2008). Hybridization was performed at $50^{\circ} \mathrm{C}$ for $36 \mathrm{~h}$ with digoxigenin (DIG)-conjugated riboprobes in prehybridization solution. The color was developed using nitroblue tetrazolium chloride (NBT) and 5-bromo-4-chloro-3'-indolyl phosphate p-toluidine salt (BCIP), the reaction was arrested by two rinses in PBS for 5 min. All samples were observed with a Nikon SMZ 1500 stereomicroscope.

\section{Relative quantitative real-time PCR}

Relative quantitative real-time PCR was used to monitor the quantitative expression of the DjStag gene essentially as described (Jiang et al., 2006) in intact and regenerating planarians at different times after cutting at the pre- and post-pharyngeal level. The cDNAs used for PCR were synthesized with the reverse transcription system using oligo d (T) primer after total RNAs were extracted using RNAiso Reagent (TaKaRa). The planarian $\beta$-actin gene was used as the internal reference gene. The PCR primers used were as follows: DjStag forward, 5'-ACACCGCCAAAATGG TTATG-3'; DjStag reverse, 5'-TGCGTTGGGAACTGTGTCTA-3'; $\beta$-actin forward, 5'-GGATG ATGAGATGCGATGTTG-3'; $\beta$-actin reverse, 5'-ATGCCAGGTCCAGATTCGTCA-3'.

For each primer/cDNA sample combination, PCRs were performed in sextuple. Relative quantitative real-time PCR was carried out on a 7500 Real-Time PCR System (Applied Biosystems, CA, USA) using a $\mathrm{SYBR}^{\circledR}$ Green Real-Time PCR Master Mix (TOYOBO) following the manufacturer protocol. Cycling parameters were: $50^{\circ} \mathrm{C}$ for 2 min, $95^{\circ} \mathrm{C}$ for $1 \mathrm{~min}$ followed by 40 cycles of $95^{\circ} \mathrm{C}$ for $15 \mathrm{~s}, 55.5^{\circ} \mathrm{C}$ for $15 \mathrm{~s}$, and $72^{\circ} \mathrm{C}$ for $1 \mathrm{~min}$. Dissociation curves were analyzed at the end of each run to determine the purity of product and specificity of amplification.

\section{RESULTS AND DISCUSSION}

\section{The expression pattern of $\mathrm{DjStag}$ in intact planarians}

To investigate the expression patterns of $\mathrm{DjStag}$ in intact planarians, whole-mount in situ hybridization was carried out with a DIG-labeled RNA probe (Figure 1). Dorsally, DjStag is expressed in cells located at the periphery of the head and tail, around the body margins (Figure 1a). Ventrally, DjStag transcripts were specifically accumulated in the head region and distributed in some cells of the parenchyma (Figure 1b). Moreover, there are more DjStag-expressing cells in the pre-pharyngeal region than in the post-pharyngeal region (Figure 1a and $\mathrm{b}$ ). It is possible that the stronger hybridization signal observed anterior to the pharynx is due to the presence of cells expressing higher level of DjStag transcripts with respect to the DjStag-positive cells located posterior to the pharynx. No DjStag hybrid- 
ization signal was observed in control specimens hybridized with a DjStag sense RNA probe (Figure 1c).
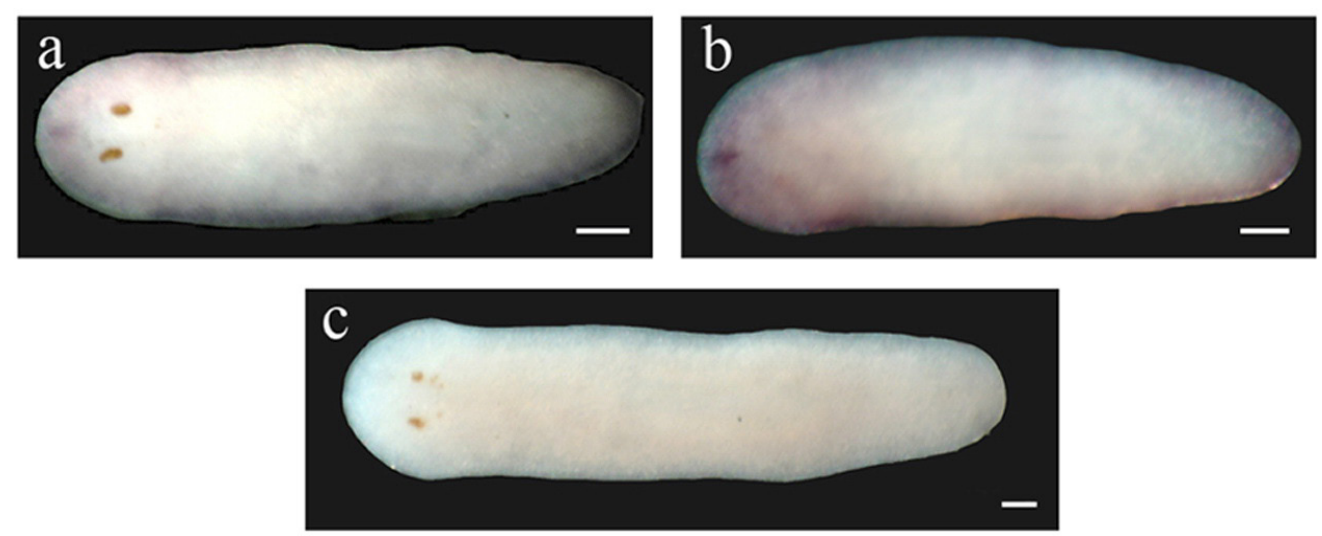

Figure 1. Expression patterns of DjStag mRNA in Dugesia japonica intact planarian detected by whole-mount in situ hybridization. a. Dorsal view. b. Ventral view. c. Dorsal view of a planarian (control) processed and hybridized similarly with DjStag sense RNA probes. No signal is seen in the control planarian. In all images, anterior is to the left. Scale bars represent $100 \mu \mathrm{M}$.

\section{The expression pattern of DjStag in regenerating planarians}

Planarians are a well-known model for studying regeneration. In order to analyze the expression pattern of the planarian DjStag gene during this process, we performed in situ hybridizations on head pieces regenerating a new posterior region (Figure 2), tail pieces regenerating a new anterior region (Figure 3 ) and on trunk pieces regenerating a new anterior and posterior region (Figure 4). During the first few days of regeneration the blastema is clearly distinguishable from the pre-existing tissues because of its lack of pigmentation (Molina et al., 2009).

Figure 2 shows the expression pattern of DjStag in regenerates from head pieces. At 1 day of regeneration, expression is observed in the same region as in intact planarians, but there is no signal in the posterior blastema (Figure 2a and a'). As regeneration proceeds, the expression of DjStag increases in amount. The increase can be seen comparing the hybridization signal at 1 day (Figure 2a and a') with that at 3 days (Figure $2 b$ and b') after cutting. At day 3, DjStag-positive signals were detected close to the posterior blastema (Figure $2 \mathrm{~b}$ and b'). Ventrally, positive signals formed a ring around the center of the body, and some dispersed signals were also observed throughout the parenchyma (Figure 2b'). It is possible that neoblasts, distributed throughout the body and already expressing DjStag transcripts, would first migrate to the site of cutting. Cell proliferation then results in an increase in DjStag transcript levels, which are maintained through early differentiation events in the blastema. With the development of the blastema, DjStag-positive signals within the posterior blastema become evident at day 5 (Figure 2c and c'). Later, at day 7, this gene is weakly expressed in the tail of the ventral side of the body (Figure $2 \mathrm{~d}$ and $\mathrm{d}$ '). No signals were detected elsewhere. 


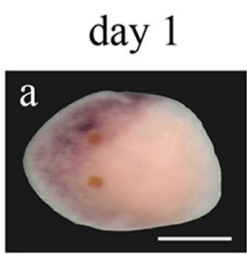

day 3
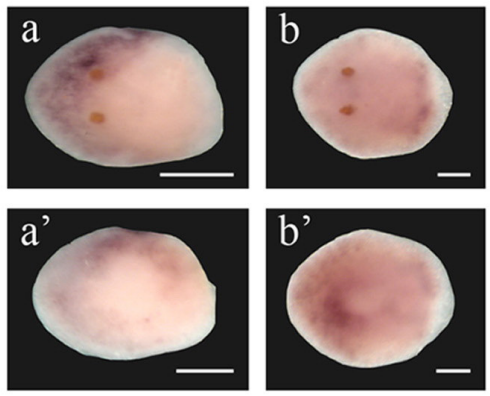

day 5
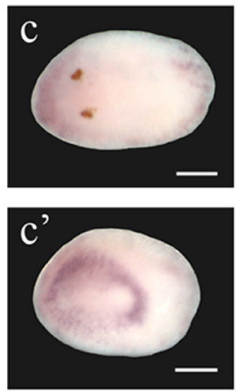

day 7
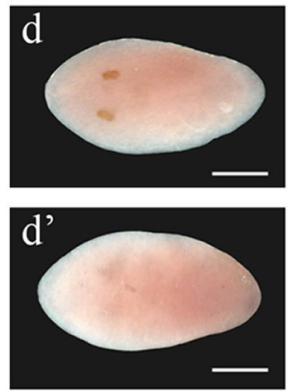

Figure 2. Expression patterns of DjStag in regenerates from head pieces. Dorsal view (upper column) and ventral view (lower column) of regenerates. a. and a'. 1-day regenerating pieces, there is no signal in the posterior blastema. b. and b'. 3-day regenerating pieces, DjStag-positive signals were detected in close to the posterior blastema. c. and c'. 5-day regenerating pieces, DjStag-positive signals within the posterior blastema become evident. d. and d'. 7-day regenerating pieces, DjStag is weakly expressed in the tail of the ventral side of the body. In all images, anterior is to the left. Scale bars represent $100 \mu \mathrm{M}$.

During anterior regeneration there is no signal in the anterior blastema as early as one day after amputation (Figure 3a and a'). By day 3, DjStag is expressed not only in the blastema, but also in the parenchyma (Figure $3 \mathrm{~b}$ and b'). At 5 days of regeneration, DjStag showed a similar pattern to that found in 3 days after regeneration (Figure $3 \mathrm{c}$ and $\left.\mathrm{c}^{\prime}\right)$. After regeneration for 7 days, the head was formed. The expression of DjStag is dramatically reduced. It weakly expressed in the head and tail (Figure 3d and d').
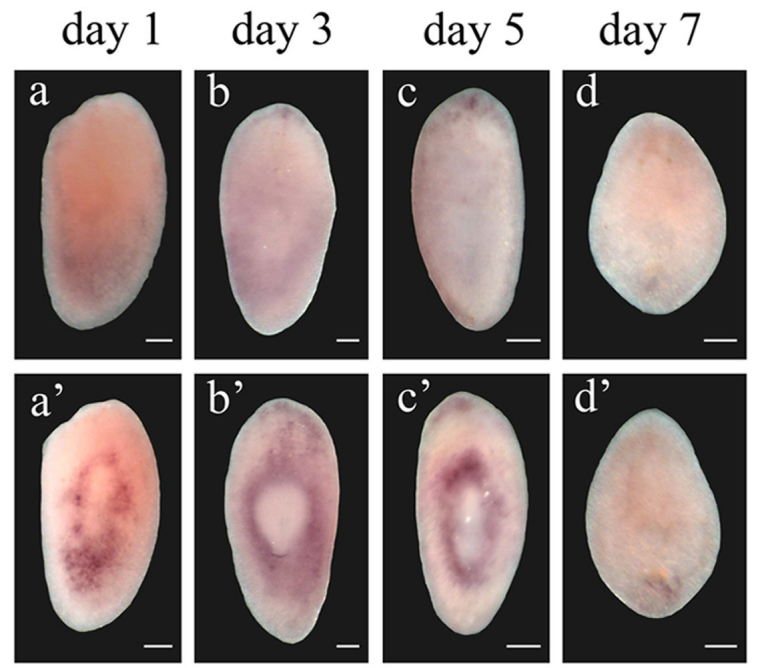

Figure 3. Expression patterns of DjStag in regenerates from tail pieces. Dorsal view (upper column) and ventral view (lower column) of regenerates. a. and a'. 1-day regenerating pieces, there is no signal in the anterior blastema. b. and b'. 3-day regenerating pieces, DjStag is expressed not only in the blastema, but also in the parenchyma. c. and c'.5-day regenerating pieces, DjStag showed a similar pattern to that found in pieces 3 days after regeneration. d. and d'. 7-day regenerating pieces. In all images, anterior is to the top. Scale bars represent $100 \mu \mathrm{M}$. 
During the early stages of regeneration from trunk pieces, no expression of DjStag is detected within the blastema; DjStag is found around the center of the stump (Figure 4a and a'). At 3 days after amputation, however, many positive signals are found within the anterior and posterior (Figure $4 \mathrm{~b}$ and b') blastemas, and distributed in a dotted pattern throughout the parenchyma of the ventral side of the body (Figure 4b'). At 5-6 days after cutting, differentiation of the missing structures becomes evident in the cephalic blastema with eyespot formation (Coward, 1968; Salvetti et al., 1998). By day 5, the signals are even more apparent and DjStag is expressed at the periphery of the forming head region (Figure 4c) and throughout the parenchyma (Figure 4c'). At day 7, this pattern of expression differs from that on the fifth day of regeneration but is nearly identical to the expression pattern observed in intact animals (Figure $4 \mathrm{~d}$ and d').
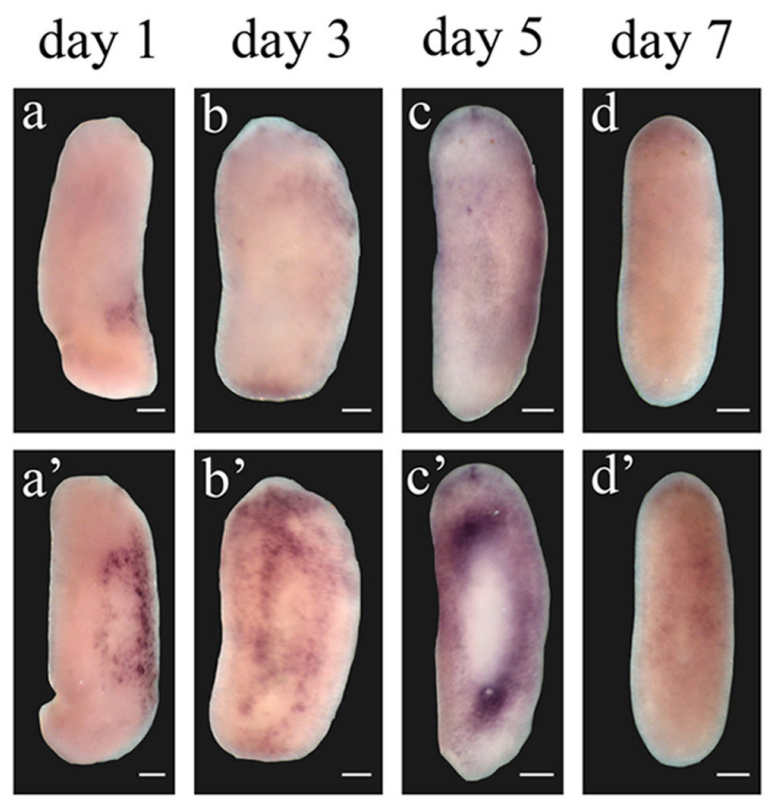

Figure 4. Expression patterns of DjStag in regenerates from trunk pieces. Dorsal view (upper column) and ventral view (lower column) of regenerates. a. and a'. 1-day regenerating pieces. b. and b'. 3-day regenerating pieces. c. and c'. 5-day regenerating pieces. d. and d'. 7-day regenerating pieces. In all images, anterior is to the top. Scale bars represent $100 \mu \mathrm{M}$.

The complete structure of planarians can be rebuilt from any piece of their body, a capacity that depends on the pluripotency of the neoblasts. With the exception of the germline, neoblasts are thought to be the only planarian cells capable of division (Baguñà et al., 1989; Wang et al., 2007). In addition to their role in regeneration, neoblasts are required for homeostasis in intact animals, where they serve to replace damaged or nonfunctional cells (Newmark and Sanchez, 2002). Regeneration in these animals follows a mixed morphallactic-epimorphic model that involves the formation of a blastema (Salo and Baguna, 1984; Salo, 2006; Adell et al., 2008). After amputation, the neoblasts adjacent to the wound proliferate, giving rise to the regenerative blastema where new structures will differentiate (Sánchez and Kang, 2005; 
Handberg-Thorsager et al., 2008). Neoblasts, distributed throughout the body and already expressing DjStag transcripts, would first migrate to the wound area. A specific localization then occurs in response to injury, that is, as a stress-related event (Salvetti et al., 1998). Cell proliferation then results in an increase in DjStag transcript levels during regeneration.

\section{Expression of DjStag is upregulated during regeneration}

Relative quantitative real-time PCR analysis was performed to investigate the change of expression of DjStag mRNA during planarian regeneration. We examined RNA samples from normal intact planarians and regenerating planarians after amputation for 1 day, 3 days, 5 days, and 7 days, respectively. Relative quantitative real-time PCR analysis of DjStag mRNA indicated that the expression of DjStag mRNA was increased after amputation compared to normal intact planarians, and the maximum level of expression of DjStag transcripts occurred at 5 days after amputation (Figure 5). The lower level of DjStag mRNA toward the end of regeneration (7 days) is similar to the basal expression level found in the mRNA of intact planarians. The result demonstrates that regeneration is required to significantly increase the expression of the DjStag. It is, therefore, probable that it plays a role in maintaining the ability of pluripotent stem cells to regenerate lost tissue in planarians.

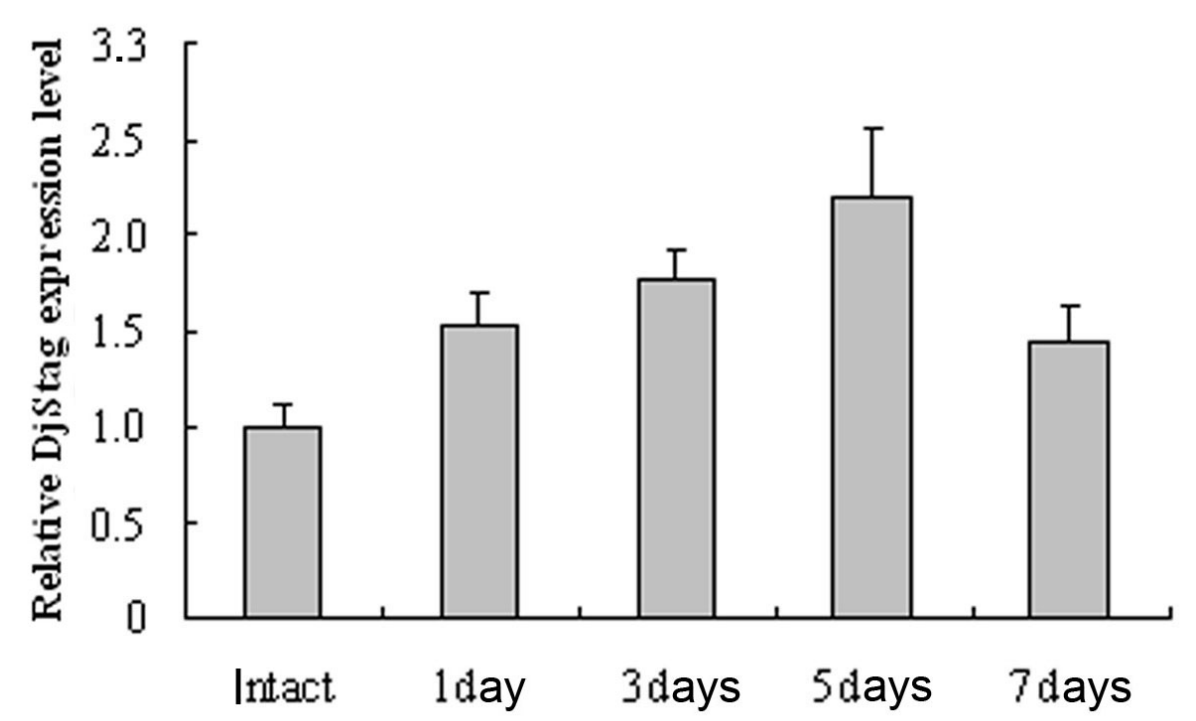

Figure 5. Expression analysis of DjStag mRNA by relative quantitative real-time PCR. Expression of DjStag mRNA was increased after amputation compared to normal intact planarians. Each gene expression level was normalized relative to the level of planarian $\beta$-actin expression. Standard deviation from the mean is indicated by error bars.

Comparable patterns are observed in the different types of regeneration resulting from the different orientations of cutting in this study. All results including regenerates from head, tail and trunk pieces showed that no expression of DjStag was detected within the blastemas at day 1 of regeneration; the first localized transcripts of DjStag were detected in the blastemas at 3 days after amputation. It would appear that the DjStag gene was activated during the stage 
of regeneration. At 3 and 5 days after amputation, positive signals formed a ring around the center of the body. Examination of many preparations indicated that the maximum level of expression of DjStag transcripts occurred at 5 days after cutting. After regeneration for 7 days, DjStag was weakly expressed. A similar decrease occurs regardless of the orientation of cut (Figures 2, 3 and 4). Furthermore, there are more DjStag-expressing cells in the ventral side than in the dorsal side during the process of regeneration (Figures 2, 3 and 4). The expression pattern does not show significant differences in the different types of regeneration. Therefore, the expression patterns between posterior, anterior, and bidirectional regenerations that have been found were similar.

During regeneration the DjStag hybridization signal reorganizes in a short time, and accumulates in the growing blastema. DjStag-positive cells appeared in the growing blastema and around the pharynx of regenerating fragments obtained from body regions that did not show DjStag hybridization signal in intact animals. This reorganization suggests that dynamic morphogenesis occurs throughout the stump (Rossi et al., 2006). It is clear from the above that DjStag transcripts show an increase, and then a decrease during regeneration. Neoblasts, might already be loaded with regulatory gene products in intact planarians, amputation serving to stimulate quantitative changes (Baguñà et al., 1994). Rapid local migration of neoblasts to the wound site, together with cell proliferation, would be sufficient to account for the upregulated hybridization seen in this study. The pattern noted by whole-mount in situ hybridization was thus consistent with the data generated by relative quantitative real-time PCR, which suggests that the DjStag implicated in planarian regeneration may play a role in maintaining the ability of pluripotent stem cells to regenerate lost tissue in planarians.

\section{ACKNOWLEDGMENTS}

We thank members of our laboratory for suggestions and encouragement. Research supported by grants from Start-up Foundation for Ph.D. in Shandong University of Technology (\#4041-410013) and Shandong Province Natural Science Foundation of China (\#ZR2009DM029).

\section{REFERENCES}

Adell T, Marsal M and Salo E (2008). Planarian GSK3s are involved in neural regeneration. Dev. Genes Evol. 218: 89-103. Anderson DE, Losada A, Erickson HP and Hirano T (2002). Condensin and cohesin display different arm conformations with characteristic hinge angles. J. Cell Biol. 156: 419-424.

Baguñà J, Saló E and Auladell C (1989). Regeneration and pattern formation in planarians III. Evidence that neoblasts are totipotent stem cells and the source of blastema cells. Development 107: 77-86.

Baguñà J, Saló E, Romero RR, Garcia-Fernandez J, et al. (1994). Regeneration and pattern formation in planarians: cell, molecules and genes. Zool. Sci. 11: 781-795.

Chen F, Kamradt M, Mulcahy M, Byun Y, et al. (2002). Caspase proteolysis of the cohesin component RAD21 promotes apoptosis. J. Biol. Chem. 277: 16775-16781.

Coward SJ (1968). Effects of actinomycin D on regeneration give evidence of sequential gene activation. Nature 219: $1257-1258$.

Gruber S, Haering CH and Nasmyth K (2003). Chromosomal cohesin forms a ring. Cell 112: 765-777.

Haering CH and Nasmyth K (2003). Building and breaking bridges between sister chromatids. Bioessays 25: 1178-1191.

Handberg-Thorsager M, Fernandez E and Salo E (2008). Stem cells and regeneration in planarians. Front. Biosci. 13: 6374-6394.

Jiang Y, Loker ES and Zhang SM (2006). In vivo and in vitro knockdown of FREP2 gene expression in the snail 
Biomphalaria glabrata using RNA interference. Dev. Comp. Immunol. 30: 855-866.

Krasikova A, Barbero JL and Gaginskaya E (2005). Cohesion proteins are present in centromere protein bodies associated with avian lampbrush chromosomes. Chromosome Res. 13: 675-685.

Lara-Pezzi E, Pezzi N, Prieto I, Barthelemy I, et al. (2004). Evidence of a transcriptional co-activator function of cohesin STAG/SA/Scc3. J. Biol. Chem. 279: 6553-6559.

Losada A, Hirano M and Hirano T (1998). Identification of Xenopus SMC protein complexes required for sister chromatid cohesion. Genes Dev. 12: 1986-1997.

Losada A, Yokochi T, Kobayashi R and Hirano T (2000). Identification and characterization of SA/Scc3p subunits in the Xenopus and human cohesin complexes. J. Cell Biol. 150: 405-416.

Michaelis C, Ciosk R and Nasmyth K (1997). Cohesins: chromosomal proteins that prevent premature separation of sister chromatids. Cell 91: 35-45.

Molina MD, Salo E and Cebria F (2009). Expression pattern of the expanded noggin gene family in the planarian Schmidtea mediterranea. Gene Expr. Patterns 9: 246-253.

Newmark PA and Sanchez AA (2002). Not your father's planarian: a classic model enters the era of functional genomics. Nat. Rev. Genet 3: 210-219.

Palakodeti D, Smielewska M, Lu YC, Yeo GW, et al. (2008). The PIWI proteins SMEDWI-2 and SMEDWI-3 are required for stem cell function and piRNA expression in planarians. RNA 14: 1174-1186.

Pati D, Zhang N and Plon SE (2002). Linking sister chromatid cohesion and apoptosis: role of Rad21. Mol. Cell Biol. 22: 8267-8277.

Pezzi N, Prieto I, Kremer L, Perez Jurado LA, et al. (2000). STAG3, a novel gene encoding a protein involved in meiotic chromosome pairing and location of STAG3-related genes flanking the Williams-Beuren syndrome deletion. FASEB J. 14: 581-592.

Prieto I, Suja JA, Pezzi N, Kremer L, et al. (2001). Mammalian STAG3 is a cohesin specific to sister chromatid arms in meiosis I. Nat. Cell Biol. 3: 761-766.

Reddien PW and Sanchez AA (2004). Fundamentals of planarian regeneration. Annu. Rev. Cell Dev. Biol. 20: 725-757.

Reddien PW, Bermange AL, Kicza AM and Sanchez AA (2007). BMP signaling regulates the dorsal planarian midline and is needed for asymmetric regeneration. Development 134: 4043-4051.

Rossi L, Salvetti A, Lena A, Batistoni R, et al. (2006). DjPiwi-1, a member of the PAZ-Piwi gene family, defines a subpopulation of planarian stem cells. Dev. Genes Evol. 216: 335-346.

Salo E (2006). The power of regeneration and the stem-cell kingdom: freshwater planarians (Platyhelminthes). Bioessays 28: 546-559.

Salo E and Baguna J (1984). Regeneration and pattern formation in planarians. I. The pattern of mitosis in anterior and posterior regeneration in Dugesia (G) tigrina, and a new proposal for blastema formation. J. Embryol. Exp. Morphol. 83: $63-80$

Salvetti A, Batistoni R, Deri P, Rossi L, et al. (1998). Expression of DjY1, a protein containing a cold shock domain and RG repeat motifs, is targeted to sites of regeneration in planarians. Dev. Biol. 201: 217-229.

Sanchez AA and Kang H (2005). Multicellularity, stem cells, and the neoblasts of the planarian Schmidtea mediterranea. Exp. Cell Res. 306: 299-308.

Sjogren C and Nasmyth K (2001). Sister chromatid cohesion is required for postreplicative double-strand break repair in Saccharomyces cerevisiae. Curr. Biol. 11: 991-995.

Sumara I, Vorlaufer E, Gieffers C, Peters BH, et al. (2000). Characterization of vertebrate cohesin complexes and their regulation in prophase. J. Cell Biol. 151: 749-762.

Wang Y, Zayas RM, Guo T and Newmark PA (2007). Nanos function is essential for development and regeneration of planarian germ cells. Proc. Natl. Acad. Sci. U. S. A. 104: 5901-5906. 